\title{
A shared target
}

DOI:

10.1038/nrd2387
Chronic inflammation is associated with obesity and is implicated in the pathogenesis of insulin resistance and type 2 diabetes. Research reported in Nature demonstrates that inhibition of a protein known to integrate inflammatory and metabolic responses - the adipocyte fatty-acidbinding protein (Ap2; also known as FABP4) - may be a novel strategy for the treatment of diseases of the metabolic syndrome.

Ap2, which is highly expressed in adipocytes, is regulated by peroxisome-proliferator-activated receptor- $\gamma$ (PPAR $\gamma$ ) agonists, insulin and fatty acids. Deletion of the Ap2 gene in mice has indicated a dominant role for this molecule in several chronic metabolic diseases, raising the possibility of targeting Ap2 to treat such conditions.

To investigate this potential therapeutic strategy, Hotamisligil and colleagues used the small molecule BMS309403 - an orally active, potent and selective inhibitor of Ap2, which interacts with the fatty-acidbinding pocket within the interior of Ap2 - to inhibit binding of endogenous fatty acids. Using both mouse and cell-based studies, they focused on the effects of this compound in two chronic metabolic diseases: atherosclerosis and type 2 diabetes.

To study the potential of Ap2 inhibition in the treatment of atherosclerosis, the authors used the apolipoprotein E-deficient $\left(\right.$ Apoe $\left.^{-/-}\right)$ mouse model of this disease. In mice fed on a western diet, $A p o e^{-/-}$ mice treated with BMS309403 had a reduced extent of atherosclerotic lesions in the proximal aorta compared with vehicletreated mice. Such lesions are almost exclusively comprised of foam cells derived from macrophages, a process regulated by the fatty-acid-binding proteins. Cell-based studies designed to identify the mechanism of action of BMS309403 revealed reduced formation of macrophage-derived foam cells, together with decreased cholesterol ester accumulation, increased cholesterol efflux and decreased production of several inflammatory mediators.

Next, to assess the potential of Ap2 inhibition in the treatment of type 2 diabetes, the effects of BMS309403 in genetic (leptindeficient ob/ob; Lep $p^{o b / o b}$ ) and diet-induced mouse models of obesity and insulin resistance were investigated. Inhibition of Ap2 improved glucose metabolism and enhanced insulin sensitivity in both mouse models. Furthermore, in $o b / o b$ mice the expression of obesityassociated inflammatory mediators was suppressed in adipose tissue in conjunction with decreased fatty liver infiltration. Interestingly, the activity of obesity-induced c-Jun N-terminal kinase 1 (JNK1; also known as MAPK8) - which is critical in the generation of inflammatory responses and inhibition of insulin action - was attenuated together with improved insulin sensitivity in the liver and adipose tissue.
Importantly, confirmation of a specific role of Ap2 inhibition in mediating the beneficial effects of BMS309403 was demonstrated by the lack of effect of this compound in macrophages and adipocytes lacking Ap2; both of which could be rendered responsive following re-expression of Ap2. Previous studies suggest that the expression, regulation and metabolic function of human Ap2 may be similar to that of mice, and so chemical inhibitors of Ap2 in humans might become a promising new class of potential drugs for the prevention and treatment of metabolic diseases.

\section{Sarah Crunkhorn}

ORIGINAL RESEARCH PAPER Furuhashi, M. et al. Treatment of diabetes and atherosclerosis by inhibiting fatty-acid binding protein aP2. Nature 447, 959-965 (2007) 